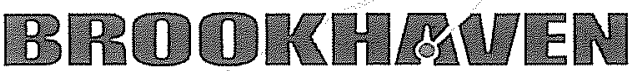 \\ NATIONAL LABORATORY
}

BNL-90259-2009-CP

\section{Proliferation Resistance and Physical Protection Evaluation Methodology Development and Applications}

\author{
$\mathbb{R}$. Bari ${ }^{1}, \mathbb{P}$. Peterson ${ }^{2}, \mathbb{I}$. Therios ${ }^{3}$, J. Whitlock ${ }^{4}$ \\ 'Brookhaven National Laboratory, Upton, NY 11973, USA \\ ${ }^{2}$ Department of Nuclear Engineering, University of California, \\ Berkeley, CA 94720-1730, USA \\ ${ }^{3}$ Argonne National Laboratory, Argonne, IL 60439, USA \\ *Atomic Energy of Canada Limited, Chalk River Laboratories, Chalk River, \\ Ontario, Canada, KOJ IJO
}

To be presented at the GIF Symposium

Paris, France, September 9-10, 2009

July 2009

\author{
Energy Sciences and Technology Department \\ Brookhaven National Laboratory \\ P.O. Box 5000 \\ Upton, NY 11973-5000 \\ www.bnl.gov
}

\begin{abstract}
Notice: This manuscript has been authored by employees of Brookhaven Science Associates, LLC under Contract No. DE-AC02-98CH10886 with the U.S. Department of Energy. The publisher by accepting the manuscript for publication acknowledges that the United States Government retains a non-exclusive, paid-up, irrevocable, world-wide license to publish or reproduce the published form of this manuscript, or allow others to do so, for United States Government purposes
\end{abstract}


This preprint is intended for publication in a journal or proceedings. Since changes may be made before

publication, it may not be cited or reproduced without the author's permission

\section{DISCLAIMER}

This report was prepared as an account of work sponsored by an agency of the United States Government. Neither the United States Government nor any agency thereof, nor any of their employees, nor any of their contractors, subcontractors, or their employees, makes any warranty, express or implied, or assumes any legal liability or responsibility for the accuracy, completeness, or any third party's use or the results of such use of any information, apparatus, product, or process disclosed, or represents that its use would not infringe privately owned rights. Reference herein to any specific commercial product, process, or service by trade name, trademark, manufacturer, or otherwise, does not necessarily constitute or imply its endorsement, recommendation, or favoring by the United States Government or any agency thereof or its contractors or subcontractors. The views and opinions of authors expressed herein do not necessarily state or reflect those of the United States Government or any agency thereof. 


\title{
PROLIFERATION RESISTANCE AND PHYSICAL PROTECTION EVALUATION METHODOLOGY DEVELOPMENT AND APPLICATIONS
}

\author{
Robert A. Bari \\ Brookhaven National Laboratory \\ PO Box 5000 Upton, NY 11973-5000 USA \\ Tel: 631-344-2629, Fax: 631-344-5957,Email:bari@bnl.gov \\ Per F. Peterson \\ Department of Nuclear Engineering \\ University of California, Berkeley, CA 94720-1730 USA \\ Tel: 510-643-7749, Fax: 510-643-968.5, Email.peterson@nuc.berkeley.edu \\ Ike U. Therios \\ Argonne National Laboratory \\ 9700 S. Cass Avenue \\ Argonne, IL 60439, USA \\ Tel: 630-2.52-7657, Fax: 630-252-4978, Email: itherios@anl.gov \\ Jeremy J. Whitlock \\ Atomic Energy of Canada Limited, Chalk River Laboratories \\ Chalk River; Ontario, Canada KOJ IJ0 \\ Tel: 613-584-8811, Email: whitlocki@aed.ca
}

\begin{abstract}
We present an overview of the technical progress and accomplishments on the evaluation methodology for proliferation resistance and physical protection (PR\&PP) of Generation IV muclear energy systems. We intend the results of the evaluations performed with the methodology for three types of users: system designers, program policy makers, and external stakeholders. The PR\&PP Working Group developed the methodology through a series of demonstration and case studies. Over the past few year's various national and international groups have applied the methodology to nuclear energy' system design as well as to developing approaches to advanced safeguards.
\end{abstract}

I. Introduction

We present the technical progress and accomplishments on the evaluation methodology for proliferation resistance and physical protection (PR\&PP) of advanced nuclear energy systems (NESs). The Generation IV Roadmap [1] recommended the development of an evaluation methodology to define measures for PR\&PP and to develop a methodology for evaluating them for the six NESs proposed within the Gen IV program. Accordingly, the Generation IV International Forum (GIF) formed a Working Group in December 2002 to develop a methodology. GIF approved the current version of the methodology (Revision 5) for open distribution and it is available at the GIF website [2].
For a proposed NES design, the methodology defines a set of challenges, analyzes system response to these challenges, and assesses outcomes. The challenges to the NES are the threats posed by potential actors (proliferant States or sub-national adversaries). The characteristics of Generation IV systems, both technical and institutional, are used to evaluate the response of the system and to determine its resistance against proliferation threats and robustness against sabotage and terrorism threats. The outcomes of the system response are expressed in terms of a set of measures, which are the high-level PR\&PP characteristics of the NES. The methodology is organized to allow evaluations to be performed at the earliest stages of system design and to become more detailed and more representative as the design progresses. It can thus be used to enable a program in safeguards by design or to enhance 
the conceptual design process of an NES with regard to intrinsic features for PR\&PP. We intend the results of the evaluations performed with the methodology for three types of users: system designers, program policy makers, and external stakeholders.

II. Objectives and Overview of Assessment Approach The Technology Goals for Generation IV nuclear energy systems (NESs) highlight Proliferation Resistance and Physical Protection (PR\&PP) as one of the four goal areas along with Sustainability, Safety and Reliability, and Economics:

Generation IV muclear energy systems will increase the assurance that they are a very unatractive and the least desirable route for diversion or theft of weapons-usable materials, and provide increased physical protection against acts of terrorism.

We define PR\&PP as follows:

Proliferation resistance is that characteristic of an NES that impedes the diversion or undeclared production of nuclear material or misuse of technology by the Host State seeking to acquire nuclear weapons or other nuclear explosive devices.
- Maintain configuration control over the PR\&PP methodology, its documentation and revisions, and serve as a central authority to review and accept methodology improvements and incorporate them in the configuration-controlled GIF PR\&PP methodology;

- Strengthen the link with Generation IV system designers, in particular with GIF System Steering Committees;

- Maintain cognizance of and interactions with other GIF-related activities, such as the Risk and Safety Working Group;

- Maintain cognizance of and interactions with non-GIF activities such as IAEA initiatives and specific national initiatives:

- Promote and facilitate early consideration of PR\&PP in the development and design of Generation IV systems;

- Promote PR\&PP goals and broad acceptance of the PR\&PP methodology by participation in conferences and publication of papers;

- Maintain capability to perform or direct PR\&PP studies on request of GIF.

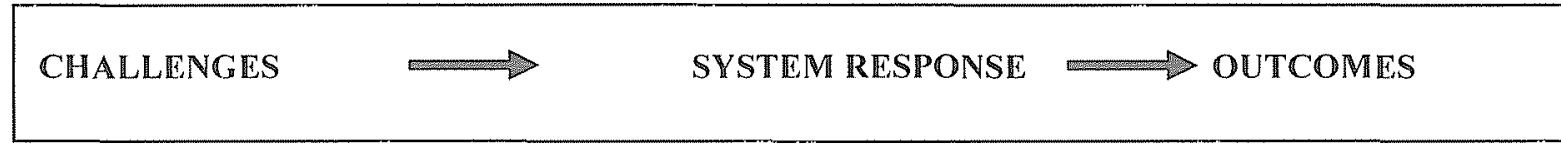

Threats

Physical protection (robustmess) is that characteristic of an NES that impedes the theft of materials suitable for nuclear explosives or radiation dispersal devices (RDDs) and the sabotage of facilities and transportation by sub-national entities and other non-Host State adversaries.

According to the current Terms of Reference approved by GIF, the responsibilities of the PR\&PP Working Group (WG) are as follows:

- Maintain cognizance of PR\&PP evaluations conducted under the auspices of GIF or with the knowledge and counsel of GIF through its member states, and serve as a clearinghouse for advice to the GIF Policy and Experts Groups on PR\&PP issues related to Generation IV nuclear energy systems;

- Monitor the integrity and quality of evaluations conducted under the auspices of GIF or with the knowledge and counsel of GIF through its member states under terms and conditions that protect proliferation-sensitive and proprietary information, provide peer-review of PR\&PP evaluations upon request, and address questions related to the fidelity with which the methodology is applied;

\section{$P R \& P P$}

Assessment

The diagram shown here illustrates the methodological approach at its most basic. As noted in the Introduction, for a given system, analysts define a set of challenges, analyze system response to these challenges, and assess outcomes.

The evaluation methodology assumes that an NES has been at least conceptualized or designed, including both the intrinsic and extrinsic protective features of the system. Intrinsic features include the physical and engineering aspects of the system; extrinsic features include institutional aspects such as safeguards and external barriers. A major thrust of the PR\&PP evaluation is to elucidate the interactions between the intrinsic and the extrinsic features, study their interplay, and then guide the path toward an optimized design.

The structure for the PR\&PP evaluation can be applied to the entire fuel cycle or to portions of an NES. The methodology is organized as a progressive approach to allow evaluations to become more detailed and more representative as system design progresses. PR\&PP evaluations should be performed at the earliest stages of design when flow diagrams are first developed in order to systematically integrate proliferation resistance and 
physical protection into the designs of Generation IV NESs along with the other high-level technology goals of sustainability, safety and reliability, and economics. This approach provides early, useful feedback to designers, program policy makers, and external stakeholders from basic process selection (e.g., recycling process and type of fuel), to detailed layout of equipment and structures, to facility demonstration testing.

\section{Recent Accomplishments}

The PR\&PP WG has recently performed a case study on an example sodium fast reactor (ESFR) and its associated fuel cycle to exercise the methodology and to obtain preliminary insights on the PR\&PP aspects of this system [3]. There is also an ongoing effort [4] to seek harmonization between the PR\&PP methodology and an initiative by the International Atomic Energy Agency on a related approach to proliferation resistance that has been developed under the International Project on Innovative Nuclear Reactors and Fuel Cycles (INPRO). The purpose of this harmonization activity is to more fully understand and articulate the range of applicability and the potential for appropriate synergy and cooperation among the two efforts. Further, the PR\&PP WG and the System Steering Committees (SSCs) for each of the six design concepts within GIF have undertaken a focused effort integrate PR\&PP notions into the design activities for each of the six concepts.

\section{Example Sodium Fast Reactor Case Study}

The PR\&PP WG has developed its methodology with the aid of a series of studies. The ESFR consists of four sodium-cooled fast reactors of medium size co-located with an on-site dry fuel storage facility and a pyrochemical spent fuel reprocessing facility.

The objectives of the Case Study were to exercise the GIF PR\&PP methodology for a complete Gen-IV reactor/fuel cycle system; to demonstrate, via the comparison of different design options, that the methodology can generate meaningful results for designers and decision makers; to provide examples of PR\&PP evaluations for future users; to facilitate transition to other studies; and to facilitate other ongoing collaborative efforts (e.g., INPRO) and other national efforts.

Lessons learned were that each PR\&PP evaluation should start with a qualitative analysis allowing scoping of the study, of the assumed threats and identification of targets, system elements, etc.; that there is a need to include detailed guidance for qualitative analyses in methodology; that the role of experts is essential; that there is a need for PR and PP experts and expert elicitation techniques; and that qualitative analysis offers valuable results, even at the preliminary design level. Qualitative analysis can directly address the measures for PR: Technical Difficulty (TD), Proliferation Time (PT), Proliferation Cost (PC), and Material Type (MT). However, Detection Resource Efficiency (DE) and especially Detection Probability (DP) are harder to quantify using qualitative analysis.

Systematic identification of potential diversion pathways is a key goal. We found that it is possible to systematically identify targets and potential pathways for each specific threat, and to systematically search for plausible scenarios that could implement the potential proliferant Host State's strategies to divert the target material. A set of diversion pathway segments were developed and the proliferation resistance measures for each pathway were determined. The methodology compares and distinguishes how different design choices affect proliferation resistance.

The diversion pathways analysis provides a variety of useful information to stakeholders, including regulatory authorities, government officials, and system designers. This information includes how attractive the material is to potential proliferators for use in a weapons program; how difficult it would be to physically access and remove the material; and whether the facility can be designed and operated in such a manner that all plausible acquisition paths are impeded by a combination of intrinsic features and extrinsic measures.

The misuse pathways analysis requires consideration of potentially complex combinations of processes to produce weapons-usable material, i.e., it is not a single action on a single piece of equipment, but rather an integrated exploitation of various assets and system elements. We found that, given a proliferation strategy, some measures are likely to dominate over the others, and within a measure some segments will dominate the overall pathway estimate.

The breakout pathways analysis found that breakout is a modifying strategy within the diversion and misuse threats and can take various forms that depend upon intent and aggressiveness, and ultimately the proliferation time assumed by a proliferant state. Furthermore, measures can be assessed differently within the breakout threat, depending upon the breakout strategy chosen. Some additional factors related to global response and foreign policy were identified as being relevant to the breakout threat, but those factors are not included in the PR\&PP methodology.

The theft and sabotage pathways analysis found that multiple target and pathways exist. The most attractive theft target materials appeared to be located in a few target areas. Specifically, for the ESFR, the most attractive theft target areas with the most attractive target materials were found to be the LWR spent fuel cask parking area, the 
LWR spent fuel storage and fuel cycle facility staging/washing area, the fuel cycle facility air cell (hot cell), and the inert hot cell.

As noted in the PR\&PP methodology report [2], a substantial base of analytic tools already exists for theft and sabotage pathway analysis. The case study verified that these tools can be used within the paradigm of the PR\&PP methodology.

The Case Study indicated that the methodology could be improved by:

- Applying the measures to a broader range of targets and pathways to gain additional experience with their practical application,

- Investigating the specific form of the metrics used to express the measures.

Interactions with Nuclear Energy System Designers

As part of the effort to familiarize GIF participants with the PR\&PP methodology, particularly system designers and program policy makers and to better understand the needs of the designers, a series of workshops were held beginning in the US in 2005, Italy in 2006, Japan in 2006, and Republic of Korea in 2008. Useful mutual information exchange occurred during these workshops which helped to further define the methodological approach and the needs of the users.

Also, in 2007 informal discussions began between the PR\&PP WG and representatives of the GIF System Steering Committees (SSCs) for each of the six Gen IV design concepts on the exploration of ways that the two entities could cooperate in the assessment and enhancement of PR\&PP performance of Gen IV systems. A workshop of interested parties was held in May 2008 at Brookhaven National Laboratory which resulted in a program plan for future joint activities. Three broad goals were defined for future joint activities: 1) identify in the near term salient features of the design concepts that impact their PR\&PP performance, 2) perform crosscutting studies that assess against PR\&PP criteria design or operating features common to various Gen IV systems, and 3) infer functional requirements for the global layout of future nuclear energy systems. See paper by F. Carre and S. Felix, Proceedings of Global 2009, for further details [5].

As of this writing, draft white papers on the PR\&PP aspects and issues each of the six design concepts are in development between representatives of the SSCS and the PR\&PP WG A follow-on workshop is planned for July 2009 to further advance the white papers and to continue future joint activities
Interactions with GIF RSWG

In addition to the establishment of the PR\&PP WG, the GIF has recognized the need for a Risk and Safety Working Group (RSWG) to address the approach to be adapted to safety of future nuclear energy systems. The GIF also recognized that an interface with the activities of the PR\&PP WG would be needed, and thus noted:

- A need for integrated consideration of safety, reliability, proliferation resistance and physical protection approaches in order to optimize their effects and minimize potential conflicts between approaches.

- A need for mutual understanding of safety priorities and their implementation in PR\&PP and RSWG evaluation methodologies.

The efforts of these two groups continue to be carefully coordinated. This has been largely accomplished so far via the close working relations between the leaderships of the two groups. Advances by either group have relevance to the other and are mutually beneficial to both. It also continues to be important to assess and understand the impact of all specific design features in relation to objectives of safety performance, physical protection, and proliferation resistance.

See Khalil et al., Proceedings of Global 2009 for further details [6].

Proliferation Risk Reduction Assessments

Assessments of proliferation risk reduction are being conducted in various countries that participate in GIF as part of their respective national programs on future options for nuclear energy. For example, in January 2009, the U.S. Department of Energy (DOE) National Nuclear Security Administration (NNSA) released a draft Non-Proliferation Impact Assessment (NPIA) of the Global Nuclear Energy Partnership (GNEP) for public comment [7]. The draft NPIA analyzes the U.S. domestic nuclear fuel alternatives identified in the draft GNEP Programmatic Environmental Impact Statement (PEIS) for their potential impacts on the risk of nuclear proliferation and on U.S. nonproliferation goals. For details on the PEIS, see http://nuclear.gov/peis.html.

In evaluating the proliferation risk associated with the GNEP fuel cycle alternatives, the NPIA considered both policy and technical factors [8]. The policy evaluation drew on the relevant objectives of U.S policy, which include discouraging the spread of enrichment and reprocessing technology, minimizing stocks of separated plutonium, promoting proliferation resistant technology, and improving international safeguards. The technical evaluation drew on the PR\&PP methodology [2]. The draft 
NPIA concluded that recycling of spent fuel may offer opportunities for the United States to discourage the spread of enrichment and reprocessing technologies by participating in comprehensive nuclear fuel services. However, the NPIA also noted that, by separating relatively attractive materials from spent fuel, such recycling also involves new risks compared to the current once-through fuel cycle.

An Element of the Next Generation Safeguards Initiative (NGSI)

International safeguards are a central pillar of the nuclear nonproliferation regime. Administered by the International Atomic Energy Agency (IAEA), international safeguards serve to monitor nuclear activities under the NonProliferation Treaty (NPT) and are the primary vehicle for verifying compliance with peaceful use and nuclear nonproliferation undertakings.

The Department of Energy's NNSA undertook a broad review of international safeguards, which concluded that a comprehensive initiative to revitalize the international safeguards technology and human resource base by leveraging U.S. technical assets and partnerships was urgently needed to keep pace with demands and emerging safeguards challenges.

To address these challenges, NNSA launched the NGSI [9] to develop the policies, concepts, technologies, expertise, and infrastructure necessary to sustain the international safeguards system as its mission evolves over the next 25 years

The deployment of new types of reactors and fuel cycle facilities, combined with the need to make the most effective and efficient use of limited safeguards resources, requires new concepts and approaches. The program plan for the NGSI calls for using the PR\&PP methodology to evaluate new nuclear system designs for proliferation risk reduction. This will be helpful in establishing a global norm for designers to systematically identify tradeoffs and evaluate and compare different options. At the same time the methodology applications would have to be of sufficient quality to avoid unwarranted reductions in safeguards and physical protection efforts

\section{Safeguards by Design}

There are ongoing and planned efforts both nationally [9] and internationally [10] to promote and implement the concept of safeguards by design (SBD) in the nuclear facility design process. These are very promising initiatives which can lead to effective and efficient introduction of safeguards early in the design process. Assessments of the benefits of SBD can be performed in the broader proliferation resistance framework. This is because, a gauge for how much proliferation risk reduction is being achieved in a SBD activity is needed to be able to understand its relative value with regard to economic, operational, safety, and security factors. An overarching PR\&PP framework would help to guide effective and efficient safeguards in the design process.

\section{Towards Harmonization with INPRO}

In parallel with the multilateral effort by GIF PR\&PP WG, and over the same time period, the International Atomic Energy Agency (IAEA) has been sponsoring development of an International Project on Innovative Nuclear Reactors and Fuel Cycles (INPRO) to help to ensure that nuclear energy is available in the $21^{\text {st }}$ century in a sustainable manner. See Pomeroy et al. [4] for additional information. In particular, INPRO has put forth basic principles, user requirements, and criteria for future nuclear energy systems, with similar broad goal areas to those that are being considered by GIF, including proliferation resistance and physical protection.

The INPRO approach [11] is primarily designed for nuclear energy system users (and thus guides the INPRO assessor in confirming that adequate proliferation resistance has been achieved in the nuclear energy system under consideration), but it can also give guidance to the developer of nuclear technology on how to improve proliferation resistance. The INPRO proliferation resistance approach identifies a Basic Principle of Proliferation Resistance and five User Requirements for meeting this Principle, along with seventeen indicators with specific criteria and acceptance limits.

The approaches share certain similarities, beginning with a common definition of proliferation resistance. Both approaches have a hierarchal analytical structure involving proliferation resistance principles, high-level evaluation factors and multiple measures or criteria related to each high-level factor. Both approaches treat proliferation resistance as a function of multiple extrinsic measures (e.g. safeguards, etc.) and intrinsic features (e.g. material attractiveness, etc.), and characterize proliferation resistance in terms of each. Both approaches recognize the concept of barriers to proliferation, but implement the concept differently. Neither approach aggregates its results into a single numerical value or grade, so that strengths and weaknesses under each of the main evaluation criteria are explicitly considered. Both approaches are primarily technical evaluations that incorporate institutional and policy contexts for the systems under consideration.

There are several notable differences between the two approaches. The INPRO approach focuses on the proliferation resistance of a declared, safeguarded nuclear energy system in a specific State, and implicitly excludes from the analysis clandestine facilities (including those that 
might be needed to complete a proliferation pathway) or a breakout scenario (in which a facility is overtly misused for proliferation purposes). In comparison, the GIF approach considers both declared and undeclared facilities and activities, to complete the proliferation pathway from acquisition and processing of material to fabrication of a nuclear explosive device as well as overt misuse following breakout.

\section{Future Directions}

As the world increases its use and reliance on nuclear technologies for energy and other peaceful applications, there will be a need for a corresponding effort to assure that nonproliferation goals, as enunciated by the IAEA, are realized. There are many national and international programs that are aimed at providing this assurance. The PR\&PP methodology is an analysis tool that can help to assess and manage the risks posed by threats to the peaceful use of nuclear technologies. Some area in which PR\&PP studies could prove effective in reducing proliferation risk are indicated below.

As new and innovative design are developed for nuclear energy systems through GIF and INPRO, the PR\&PP methodology approach will be essential to incorporating good design principles for proliferation resistance and physical protection into new emerging and viable concepts. The work that is just beginning between the PR\&PP WG and the GIF SSCs will serve as a key model for how to implement this process. The PR\&PP WG is in the early stages of planning a follow-on case study to the one recently completed on the example sodium fast reactor. Consideration is being given to a case study on a very high temperature gas-cooled reactor.

The PR\&PP methodology approach can be a useful tool in developing safeguards by design as outlined in the Next Generation Safeguards Initiative and in recent parallel activities by the 1AEA. Results of PR\&PP evaluations can serve as clear discriminators among design altematives and could thus help to make choices that reduce proliferation risk.

The PR\&PP methodology can be used to evaluate the proliferation impacts associated with particular cases of export of nuclear fuel cycle technologies, materials, and information or to address the broader issue of evaluating the effectiveness of current practices.

\section{Conclusions}

The GIF PR\&PP evaluation methodology was initially motivated by the need to have an approach to the assessment of new nuclear energy design concepts that were envisioned within the GIF program. The methodology that has been developed now enjoys wide international consensus and has been used in applications beyond the initial purpose. It is expected that subsequent applications of the methodology will 1) lead to refinement of the approach which will streamline and focus it to address issues of interest to end-users of the results and 2) have application to a more diverse set of applications that will enhance decision making in the PR\&PP areas.

\section{ACKNOWLEDGMENTS}

The efforts and ideas of the many members of the PR\&PP working group over several years is the foundation of this summary paper. The sponsorship of the organizations within the participating GIF countries is gratefully acknowledged.

\section{REFERENCES}

[1] DOE (U.S. Department of Energy), Nuclear Energy Research Advisory Committee and the Generation IV International Forum. December 2002. A Technology Roadmap for Generation IV Nuclear Energy Systems. GIF002-00, DOE Nuclear Energy Research Advisory Committee and the Generation IV International Forum, Washington, D.C.

[2] "Evaluation Methodology for Proliferation Resistance and Physical Protection of Generation IV Nuclear. Energy Systems," Revision 5, Generation IV International Forum, GIF/PRPPWG/2006/005, November30,2006;www.gen4.org/Technology/horizon tal/PRPPEM.pdf. A summary of this methodology by P. Peterson appears in these Proceedings.

[3] Panel Sessions Presentations on Proliferation Resistance and Physical Protection of Generation IV Nuclear Energy Systems at Global 2007 Conference, Sponsored by the American Nuclear Society, Boise, Idaho, September 9-13, 2007. Also, for the results of this Case Study, see these Proceedings of Global 2009, September 2009: M. Zentner et al (Diversion Threat); G. Cojazzi et al. (Misuse Threat); J. Whitlock (Breakout Threat).

[4] G. Pomeroy, E. Wonder, R. Bari, M. Zentner, "Approaches to Evaluation of Proliferation Resistance of Nuclear Systems," Proceedings of the Institute of Nuclear Materials Management, Annual Meeting, Nashville, TN, July 13-17, 2008.

[5] F. Carre, S. Felix, "Proliferation Resistance and Physical Protection in the Generation IV International Forum System Design Concepts," Proceedings of Global 2009, September 2009.

[6] H. Khalil, R. Bari, G-L. Fiorini, T. Leahy, P.F. Peterson, R. Versluis, "Integration of Safety and Reliability with Proliferation Resistance and Physical Protection for Generation IV Nuclear Energy 
Systems," Proceedings of Global 2009, September 2009.

[7] Draft Non-Proliferation Impact Assessment (NPIA) of the Global Nuclear Energy Partnership (GNEP), December2008,

nnsa.energy gov/nuclear_nonproliferation/documents $/ G N E P$ NPIA $p d f$

[8] M.W. Goodman, J.K. Sprinkle, A.M. Scheinman, "A Non-Proliferation Impact Assessment of the GNEP Alternatives," Proceedings of Global 2009, September 2009.

[9] A description of this program can be found at http://mnsa.energy.gov/nuclear nonproliferation/ nuclear safeguards.htm

[10] See Report on "Facility Design and Plant Operation Features that Facilitate the Implementation of IAEA Safeguards" Workshop conducted from October 28-31, 2008 at IAEA Headquarters in Vienna, Austria. IAEA Report STR-360, February 2009.

[11] International Atomic Energy Agency. Guidance for the Application of an Assessment Methodology for Innovative Nuclear Energy Systems, INPRO Manual Proliferation Resistance Volume 5 of the Final Report of Phase 1 of the International Project on Innovative Nuclear Reactors and Fuel Cycles (INPRO), IAEATECDOC-1575, October 2007. 